\title{
鳄龟科和平胸龟科线粒体控制区序列分析和结构比较
}

\author{
颜 亮, 张 雁, 汪 宁, 张 莉, 聂刘旺* \\ (安徽师范大学 生命科学学院, 安徽重要生物资源保护与利用研究重点实验室, 安徽 芜湖 241000)
}

摘要: 本文参照龟类近缘种的线粒体 DNA（mitochondrial DNA, mtDNA）控制区（control region, CR）及邻 接序列, 设计了二对特异引物, 采用 PCR 和测序技术, 获得了大鳄龟(Macroclemys temminckii)、小鳄龟(Chelydra serpentina)和平胸龟(Platysternon megacephalum)mtDNA CR 区序列, 其长度分别为 $1089 \mathrm{bp} 、 1124 \mathrm{bp}$ 和 $1119 \mathrm{bp}$; $\mathrm{A}+\mathrm{T}$ 的含量分别为 $68.97 \%$ 、69.34\%和 $69.44 \%$ 。序列分析显示, 三种龟 CR 区 $3^{\prime}$ 末端均存在丰富的微卫星序列, 其中大鳄龟和小鳄龟各有一段 $2 \mathrm{bp}$ 的 TA 序列分别重复 20 和 15 次; 小鳄龟另有一段 $5 \mathrm{bp}$ 的 TATAT 序列重复 13 次; 平胸龟则是一段 $10 \mathrm{bp}$ 的 AGTATGTTAT 序列重复 4 次和一段 $17 \mathrm{bp}$ 的 GTTGTTATATAACATAT 序列重复 13 次。本文还结合 GenBank 中已发表的其他 6 种龟鳖类动物的控制区序列, 探讨了龟鳖类动物微卫星序列的类 型及分布, 结果表明: 9 种龟鳖类动物都存在丰富的微卫星序列, 且微卫星所在位置及序列存在很大差异。

关键词: 大鳄龟; 小鳄龟; 平胸龟; 线粒体 DNA; 控制区; 微卫星

中图分类号: Q959.63; Q951.3; Q349 文献标识码: A 文章编号: 0254-5853-(2008)02-0127-07

\section{Comparison of Mitochondrial Control Region Sequences Between Chelydridae and Platysternidae}

\author{
YAN Liang, ZHANG Yan, WANG Ning, ZHANG Li, NIE Liu-wang* \\ (College of Life Sciences, Anhui Normal University, the Provincial Key Lab of the Conservation and Exploitation Research of Biological Resources \\ in Anhui, Wuhu Anhui 241000, China)
}

\begin{abstract}
The sequences of mitochondrial control region (CR) of Macroclemys temminckii, Chelydra serpentina and Platysternon megacephalum were obtained using PCR and sequencing techniques, with gene-specific primers, based on the CR and its flanking sequences from other species. The CR lengths of the three species were $1089 \mathrm{bp}, 1124 \mathrm{bp}$ and $1119 \mathrm{bp}$ respectively, and the base composition of A+T were $68.97 \%, 69.34 \%$ and $69.44 \%$ respectively. One fragment of (TA) $)_{20}$ microsatellite was found in M. temminckii; one fragment of (TATAT) ${ }_{13}$ direct tandem repeats followed by (TA) 15 microsatellites were found in C.serpentina; and one fragment of (AGTATGTTAT) ${ }_{4}$ direct tandem repeats followed by (GTTGTTATATAACATAT) $_{13}$ repeats were found in P. megacephalum. The distribution of mtDNA microsatellites in tortoises was discussed based on the CR sequences of the three species and other six tortoises published in GenBank. The result suggested that all the nine tortoises have microsatellites, and there are obvious differences both at the site and the sequences.
\end{abstract}

Key words: Macroclemys temminckii; Chelydra serpentina; Platysternon megacephalum; mtDNA; Control region; Microsatellite

动物线粒体 DNA（mtDNA）与核基因存在平行 进化关系, 呈母系遗传, 具有较高的突变率, 近年 来以 mtDNA 为对象研究动物的系统进化被研究者 们广泛采用 (Zardoya et al, 2003; Stuart et al, 2004;
Krenz et al, 2005)。线粒体 DNA 的控制区（control region, CR）是线粒体基因组中最长的一段非编码 区，通常位于位于 $\mathrm{tRNA}^{\mathrm{Pro}}$ 和 $\mathrm{tRNA}^{\text {Phe }}$ 基因之间， 进化速率是其它区段的 2.8一 5 倍, 具有碱基替换速

\footnotetext{
收稿日期: 2007-09-18; 接受日期:2008-03-10

基金项目：国家自然科学基金(30640048 和 30770296); 安徽省科学与技术后备人选基金项目（2006-2）；“生物环境与生态安全”安徽省高校重点实 验室基金（2006）[Supported by National Natural Science Foundation of China (30640048，30770296)]; the Reserve Candidate Fund Project of Science and Technology in Anhui Province(2006-2); the Key Lab. of Biotic Environment and Ecology Safety in Anhui Province(2006)]

*通讯作者（Corresponding author）, E-mail: 1wnie@mail.ahnu.edu.cn

第一作者简介: 颜亮 (1983-), 男, 安徽人, 硕士, 研究方向：动物细胞与分子生物学。E-mail: yane2002@126.com
} 
率快, 存在着丰富的序列变异等特点 (Zhang et al, 2000)。控制区包含有 $\mathrm{H}$-链复制起始区 $\mathrm{O}_{\mathrm{H}}$, L-链启 动子及 $\mathrm{H}$-链启动子等重要的功能元件, 对控制区结 构和功能的研究将有助于了解其 DNA 复制、转录 的机制和进化的规律 (Guo et al, 2004)。

线粒体 DNA 控制区的结构可分为三个部分: 终 止序列区（terminal associated sequences, TAS）, 中央保守区 (central conserved sequence block, CCSB）和保守序列区 (conserved sequence block, CSB) (Sbisa et al, 1997)。在 mtDNA 控制区中常存 在有许多可变数目的串联重复序列 (Variable number of tandem repeat, VNTR), 也有人称为微卫 星序列（microsatellite），且通常发生在控制区的 3' 末端(Fu et al，2006)。Kumazawa et al（1998）曾 发现动物 mtDNA $3^{\prime}$ 端是一段多串联重复序列的区 域, 它与转座、错配表达和线粒体基因异质 (heteroplasmy)相关。迄今, 国内外关于线粒体 CR 区微卫星的研究在哺乳动物、鱼类、和鸟类中已开 展了不少工作（Liu et al，2003; Guo et al，2004;

Zhang et al，2003; Randi et al，1998）。

大鳄龟 (Macroclemys temminckii) 隶属鳄龟科 (Chelydridae) 大鳄龟属 (Macroclemys), 分布于美国 中南部。小鳄龟 (Chelydra serpentina) 隶属鳄龟科鳄 龟属 (Chelydra), 分布于北美洲和中美洲。近二十 年中, 由于过度的捕杀, 鳄龟野生种群的数目逐渐 下降, 其保护生物学的研究受到广泛的关注 (Hackler et al, 2007)。平胸龟 (Platysternon megacephalum), 隶属平胸龟科 (Platysternidae) 平胸 龟属 (Platysternon), 是亚洲特产龟类, 在我国主要分 布在华中、华南等地区。迄今, 对龟鳖类动物线粒 体 CR 区的研究还很少见, 仅在非洲侧颈龟中有微 卫星 DNA 分析的报道 (Zardoya \& Meyer, 1998)。 本研究测定了上述 3 种龟的 mtDNA CR 区, 分析比 较了其特点, 并结合 GenBank 中其他龟鳖类物种线 粒体 CR 区数据, 分析了龟鳖类 CR 区微卫星序列 的特点和分布。

\section{1 材料与方法}

\section{1 实验材料}

大鳄龟标本为雌性, 编号为 NO.05071601, 小 鳄龟标本为雄性, 编号为 NO. 05071602, 均采于合 肥市野生动物园。平胸龟标本为雄性, 编号为 NO.04061201, 采自安徽黄山。

\section{2 基因组提取}

采用 Sambrook et al (2001)的方法, 取肌肉组织, 用 SDS/蛋白酶 $\mathrm{K}$ 裂解, 酚/氯仿提取总 DNA, 琼脂 糖凝胶电泳检测提取结果。

\section{3 引物设计和 PCR 扩增}

参照中华鳖 Pelodiscus sinensis (AY687385)、 锦龟 Chrysemys picta (NC_002073) 和乌龟 Chinemys reevesii（AY676201）线粒体全基因组序 列, 采用 CLUSTAL W1.8 (Thompson et al, 1994) 软件进行比对, 用 Oligo 6.0 (Rychlik \& Rychlik, 2000）软件设计可分别覆盖三种龟线粒体 CR 区序 列的两对引物。引物序列是:

Y1: $\left(5^{\prime}-3^{\prime}\right)$ : TGATACCTGCCGACTCAATGA Y2: (5'-3'): TCCCAGTTTGTGTCTTAGCAATCGT Y3: $\left(5^{\prime}-3^{\prime}\right)$ : AACCACCGTTGTATTCAACTA Y4: (5' $\left.-3^{\prime}\right)$ : CAGTTTCATTGAGTTGGCAG

$\mathrm{PCR}$ 扩增反应体积为 $25 \mu \mathrm{L}$, 含模板基因组 DNA $100 \mathrm{ng} 、 10 \times$ Buffer $2.5 \mu \mathrm{L} 、 \mathrm{MgCl}_{2}(2.5 \mathrm{~mol} / \mathrm{L}) 2.0 \mu \mathrm{L}$ 、 $\mathrm{dNTP} 1.5 \mu \mathrm{L}$ 、上下游引物各 $1 \mu \mathrm{L} 、 1 \mathrm{U}$ Taq DNA 聚 合酶（上海英骏生物技术有限公司）, 加无菌水补 足。 $\mathrm{PCR}$ 反应的循环参数为: $94^{\circ} \mathrm{C}$ 预变性 $2 \mathrm{~min}$; $94^{\circ} \mathrm{C}$ 变性 $45 \mathrm{~s}, 59.3^{\circ} \mathrm{C}$ 退火 $40 \mathrm{~s}, 72^{\circ} \mathrm{C}$ 延伸 $90 \mathrm{~s}, 35$ 个循环; $72^{\circ} \mathrm{C}$ 延伸 $10 \mathrm{~min}$ 。PCR 扩增产物用 $1.0 \%$ 琼脂糖凝胶电泳检测, 凝胶成像仪记录结果。PCR 产物经纯化试剂盒 (大连宝生工有限公司) 纯化后, 委托上海英骏生物技术有限公司 (ABI-3730 DNA 测序仪) 进行双向测序。

\section{4 序列分析}

用 BioEdit7.0 (Hell et al，1999）和 MEGA3.0 (Kumare et al，2004) 软件统计三种龟的线粒体 CR 区序列长度、碱基百分比例和序列差异百分比 信息。用 DNASIS (Version 2.5 Hitachi Software Engineering）软件，分析 CR 区微卫星序列。本文 从 GenBank 中搜索出已测出的龟鳖类线粒体全序 列, 分析其 CR 区特点, 每个科选择 $1-2$ 个代表物 种, 共 5 科 6 种 (乌龟 Chinemys reevesii、金头闭 壳龟 Cuora aurocapitata、锦龟 Chrysemys picta、中 华鳖 Pelodiscus sinensis、缅甸陆龟 Indotestudo elongata 和球动胸龟 Kinosternon odoratus）。

\section{5 序列排列}

以 $t R N A^{\text {pro }}$ 的结束和 $t R N A^{\text {phe }}$ 的起点分别作为 控制区的起点和终点, 以保守序列 F、CSB1 的起 点分别作为终止序列区、中央保守区和保守序列区 
的分界线。

\section{2 结 果}

\subsection{3 种龟线粒体控制区长度、碱基组成及序列差 异百分比}

Peng et al（2006）和 Parham et al（2006）均报 道平胸龟的线粒体基因组中存在 2 个控制区（CR I 和 CR II），其中 CR I 位于 $t R N A^{\text {pro }}$ 和 $t R N A^{\text {phe }}$ 基 因之间, CRII 位于 $t R N A^{\mathrm{Ile}}$ 和 $t R N A^{\mathrm{Gln}}$ 基因之间。 这种双控制区的形成可以用倍增-随机删除模型 (TDRL 模型) 来解释, 即 CRII 区是 CR I 区富余拷 贝随机删除而造成的 (Inoue et al, 2003; Zhang et al, 2005; Maurol et al, 2005)。因此本研究中平胸龟只 选择线粒体的 CR I 区来做分析。

三种龟 mtDNA CR 区均位于 $t R N A^{\text {pro }}$ 和 $t R N A^{\text {phe }}$ 基因之间, 表 1 比较了 3 种龟类 mtDNA CR 区的 4 种碱基组成, 可见 $\mathrm{CR}$ 区中 4 种碱基百分比和长度 相差很大，显示出龟鳖类动物线粒体控制区的高变 异性。 $\mathrm{A}+\mathrm{T}(69.25 \%)$ 平均含量明显高于 $\mathrm{G}+\mathrm{C}$ $(30.75 \%)$ ，这与其他关于控制区的研究结果是一 致的 (Liu et al, 2002)。大鳄龟和小鳄龟 mtDNA CR 区的序列差异为 $10.79 \%$, 大鳄龟与平胸龟的序列差 异为 $25 \%$, 小鳄龟和平胸龟的序列差异为 $24.60 \%$, 可见属间差异较小、科间差异明显。

\subsection{3 种龟线粒体控制区的结构比较}

图 1 和图 2 分别显示了 3 种龟 mtDNA CR 区结 构和序列的比较。根据文献 (Serb et al, 2001), 本 文识别了三种龟 mtDNA CR 区的结构: 大鳄龟、小 鳄龟和平胸龟终止序列区全长分别为 $338 \mathrm{bp} 、 311 \mathrm{bp}$ 和 $280 \mathrm{bp}$, 有终止相关序列 TACAT; 中央保守区全 长分别是 $342 \mathrm{bp} 、 341 \mathrm{bp}$ 和 $339 \mathrm{bp}$, 有保守序列 $\mathrm{F}$ （AATCACAAGAGATAAGCAAC）；保守序列区 全长分别为 $413 \mathrm{bp} 、 473 \mathrm{bp}$ 和 $531 \mathrm{bp}$, 均有三个保 守序列, 分别是 CSB1: TTAATGCTTGTTGGACATA; CSB2: GTAAACCCCCCTACCCCCC 和 CSB3: TCGTCAAACCCCTAA。在三种龟 mtDNA
CR 区的 3'末端都发现有丰富的微卫星序列, 其中 大鳄龟为 $2 \mathrm{bp}$ 的 $\mathrm{TA}$ 序列重复 20 次; 小鳄龟为 $2 \mathrm{bp}$ 的 TA 序列重复 15 次和 $5 \mathrm{bp}$ 的 TATAT 序列重复 13 次; 平胸龟为 $10 \mathrm{bp}$ 的 AGTATGTTAT 序列重复 4 次和 $17 \mathrm{bp}$ 的 GTTGTTATATAACATAT 序列重复 13 次。从微卫星特点看, 科内及属间有相似性, 也 有区别，但科间差异明显。

\subsection{9 种龟鳖类线粒体 DNA 控制区微卫星序列比 较}

在 9 种龟鳖类动物中 (表 2), CR 区长度最长 为中华鳖 $1829 \mathrm{bp}$, 最短的为乌龟 $1072 \mathrm{bp}$ 。微卫星 序列的特征主要表现为三个方面：1、微卫星序列 以 $\mathrm{TA}$ 序列重复为主。如大鳄龟、小鳄龟、乌龟、 金头闭壳龟、锦龟和鹿动胸龟。2、在位置上, 除 了中华鳖和缅甸陆龟外, 其他 7 种的微卫星序列都 位于控制区的 $3^{\prime}$ 末端。3、重复序列的长度变化较 大, 最长的为金头闭壳龟 $384 \mathrm{bp}$, 最短的为缅甸陆 龟 $22 \mathrm{bp}$ 。

\section{3 讨 论}

mtDNA CR 区的主要功能与 mtDNA 的复制、 转录、终止相关。Liu et al (2002) 对比哺乳类动物 和其他鱼类动物的 mtDNA CR 区序列, 识别了鳑鲅 鱼类的 CSB-F、CSB-E 和 CSB-D 的关键序列, 并 认为 CSB-F 序列分开终止序列区与中央保守区。 Zeng et al (2001) 认为 mtDNA 控制区中存在终止

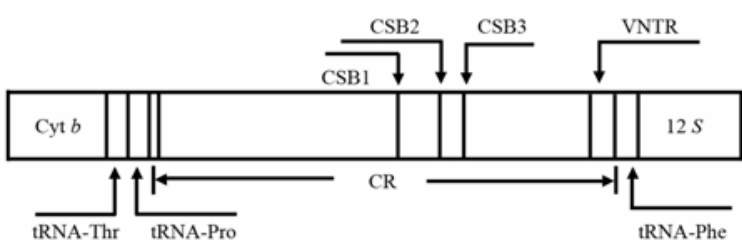

图 1 三种龟控制区结构示意图

Fig. 1 The control region structure of the three tortoises 大鳄龟有 1 个 VNTR 区, 小鲓龟和平胸龟各有 2 个 VNTR 区。 There are one VNTR in Macroclemys temminckii and two VNTR in both Chelydra serpentina and Platysternon megacephalum.

表 13 种龟线粒体 CR 区长度及四种碱基的组成

Tab. 1 Length and base composition of mtDNA control region in three tortoises

\begin{tabular}{llllllll}
\hline 物种 Species & \multicolumn{1}{c}{ 拉丁名 Latin name } & 登录号 GenBankAccession No. & A & T & G & C & 长度 Length \\
\hline 大鳄龟 & Macroclemys temminckii & EF071948 & 33.52 & 35.45 & 11.75 & 19.28 & 1089 \\
小鳄龟 & Chelydra serpentina & EF122793 & 32.26 & 37.08 & 12.03 & 18.63 & 1124 \\
平胸龟 & Platysternon megacephalum & DQ016387 & 31.81 & 37.63 & 13.89 & 16.67 & 1119 \\
\hline
\end{tabular}


Macroclemys temminckit Chelydra serpentina platysternon megacephalum

Macroclemys temminckit Chelydra serpentina platysternon megacephalum

Macroclemys temminckit Chelydra serpentina platysternon megacephalum

Macroclemys temminckit Chelydra serpentins platysternon megacephalum

Macroclemys temminckit Chelydra serpentina Platysternon megacephalum

Macroclemys temminckit Chelydra serpentina Platysternon megacephalum

Macroclemys temmincki $i$ Chelydra serpentina Platysternon megacephalum

Macroclemys temminckit Chelydra serpentina platysternon megacephalum

Macroclemys temmincki $i$ Chelydra serpentina Platysternon megacephalum

Macroclemys temminckit Chelydra serpenting platysternon megacephalum

Macroclemys temmincki $i$ Chelydra serpentina Platysternon megacephalum

Macroclemys temminckit Chelydra serpentina platysternon megacephalum

Macroclemys temminckit Chelydra serpentina platystornon mogacephalum

Macroclemys temminckit Chelydra serpentina

Platysternon megacephalum

Macroclemys temmincki $i$ Chelydra serpentina Platysternon megacephalum

Macroclemys temminckit Chelydra serpentina Platysternon megacephalum

Macroclemys temmincki $i$ Chelydra serpentina platysternon megacephalum

Macroclemys temminckii Chelydra serpentina platysternon megacephalum

Macroclemys temmincki $i$ Chelydra serpentins Platysternon megacephalum

Macroclemys temmincki $i$ Chelydra serpentins Platysternon megacephalum

Macroclemys temmincki $i$ Chelydra serpentina

platysternon megacephalum
GgAATCTTTATTTAAACTACCCTTTGACGCAGgGAATAGgCGTCC-GGCGCTTGTCGCCA ---------------------. T. . . . TA. AA. . . TT. . . . . . . . . .

TATTTACGCCCTTTTTTCTCTACCGTGCACAGTAGAGAAGTAC-TACTATAC-CACCCTA . . . T TGTT. T. . . . CT. . C. . C. . C. G. . . . . . . . . GT. . . - . . . .

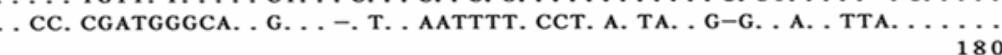
TGTATATCGTGCATTCATTTATTTACCCCTAGCATATATCTAGTAATATTACTGCTTAAT

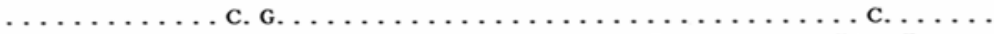
TAS TTGATTAATGACATAATATTATTGGTTTTACATAC-ATGTTCTACTTACATGACTATTAT $\ldots$ C. . . . . . . TC. . . . . . . . . . A. A. . . . . . . . .

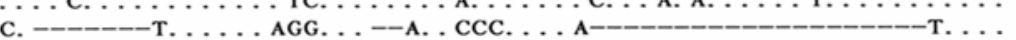
终止序列区 300

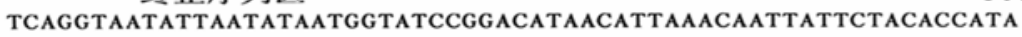
. . . . . . . . . . . A. AT. . . . . . . . . . . . . . . G CT. A. . C. G. G. T. . . A. C. AA. . . . . . . T. T. G. -GC. T. . A. . G

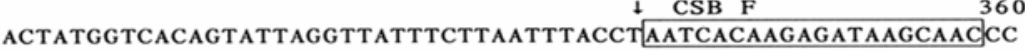
... . . . . . . . . . . . . . . . . . . . . . . . . . . . . . . $\ldots$.. . . . . . . . . . . . . . . . . . . . . . . . . . . .

TTGTTCGTAAGATACAATATCACTAGTTTCACGTCCAT-TACATTATAACGTCCATTCAT

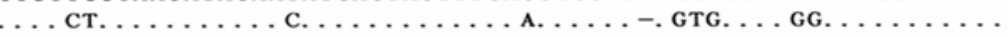

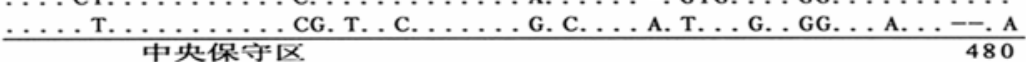
CTCTTTTTAAGAGGCCTCTGGTTGTTTTTTCAGgTACATAACATTAT-AAAGGTCATTCA

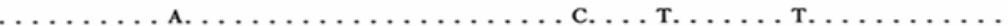
. GA. C. АTTCT. . . A. . C. . . . . A. C. . . TT. . CCACT. . T. . . G TCTCTTTTTAAAAgGCCTTTGGTTAAATGAGTTCTATACATTATATTTATAATCTGGCAT

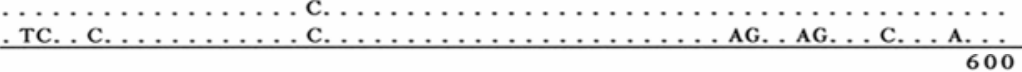

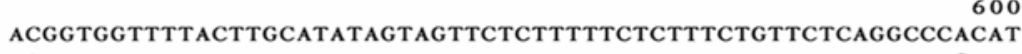

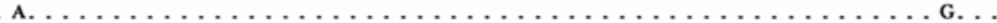
$\ldots \ldots \ldots$. . . . . . . . . . . . . . . . . . . . . . . AATTGATACCTGCCGACTCAATGAAACTGgaCTTACGTTCAAGTTGATTGgTATTACATA

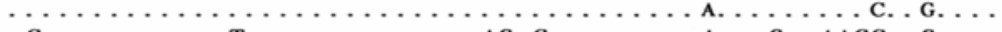
. C. . . . . . . . . . . . . AG. C. . . . . A. -. G. . AACC. . G. . ATATTGATATGGTATTATTCGATTAATGCTTGTTGGACATATACTTTTATAAAAAACCGC

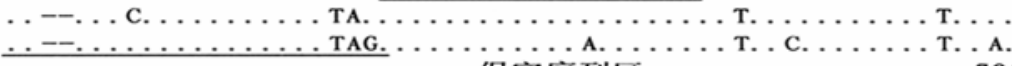
保守序列区 GACAGTAAATATAAACCTAAACGAC-AATTAAACAACTAAAAAAATAATAATTTTTAAGG $\ldots \ldots \ldots \ldots \ldots \ldots$. . T. . A. . . . . T. AT. . T. . . . . $\ldots \ldots$ T. T. . . . . АCT- . . . . . . . . . . $\operatorname{CSB} 2$ TAAACCCCCCTACCCCCCGTTAAACTAACAATTAGCCCAAATGATCACTTACTTCTCGTC $\ldots \ldots \ldots \ldots \ldots$. . . TATG. . . GC. . . . . . . . . $\mathrm{CSB} 3$ 900 AAACCCCTAAATCCGAGAGCGACCAAACTGACATAAATGCTAGTCATATAAACACTACAA ............ . . . . . . . . . . . . . . . . . T. . . A. $\ldots \ldots$. . . . . . . . . . . . T. . . . TCCTG AAAAATGAAGTATT--TATACTAGTA---CTAAAAAgTACCAAACAATTCTAAAAA--TA ... T. A. . . . . --C. . . . A. - . . . . . . . . . . . . . CA. . T. TT. . T. T. . A. GT. . C. GC. A. . AATT. . TT. T. . TAT, GTATG. . A. . GT. TGT. CACCACAT-AACCCTGACCCCTGTAT----CGTGCTACATCATACTATTTACCATTTATC . . . A. . T. G. . . . . . TCCC. .---. . A. G. . . . G. . TAC. T. T. A. . C. T. GT. TG. T. TAGTATGT. ATAC. . AGTGT. . . T. . T. . A. C. TATG. GTT. A. A. A. 1080 TTAT-ATCCTCTAC----TATATTGTATTATATTATGAATTTATATTTATTTATATATAT

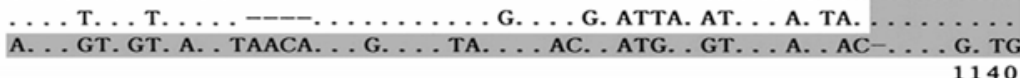

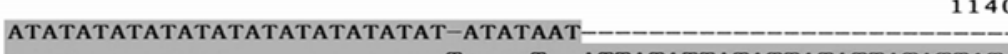
$\ldots \ldots \ldots \ldots \ldots \ldots \ldots \ldots$ T. . . . . ATTATATTATATTATATTATATTATA

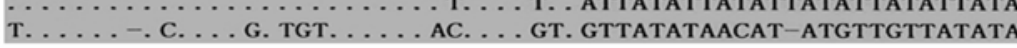
1200

TTATATTATATTATATTATATTATATAAT------------------------------ACATATGTTGTTATATAACATATGTTGTTATATAACATATGTTGTTATATAACATATGTT 1231

GTTATATAACATATGTTGTTATATAACATAT 
图 2 三种龟控制区序列比较

Fig. 2 Comparison of mtDNA control region among three tortoises

图中的点号和横杆分别表示相同的序列和缺失的序列, 方框部分标示 CSBsF、1-3 和 TAS 序列, 阴影部分表示 3' 端的重复序列, 下划线部分表示中央保守区。

Dots represent the same nucleotide as the reference sequence (at top). Dashes designate gaps that are required for insertions and deletions in one or more members of the alignment. The potential regulatory elements conserved sequence block (CSBs)F、1-3 and terminal associated sequence (TAS) are boxed. Shaded region depicts microsatellites at the $3^{\prime}$ end of control region. Underlined position represent the central conserved sequence block(CCSB).

表 29 种龟鳖类动物 mtDNA 控制区长度、微卫星基序及分布位置

Tab. 2 Length of mtDNA CR, microsatellite motif and location of nine tortoises

\begin{tabular}{|c|c|c|c|c|c|c|}
\hline 物种 Species & 拉丁名 Latin name & $\begin{array}{c}\text { 登录号 GenBank } \\
\text { Accession No. }\end{array}$ & $\begin{array}{c}\text { 微卫星基序 } \\
\text { Microsatellitemotif }\end{array}$ & $\begin{array}{c}\text { 重复次数 } \\
\text { Repeattimes }\end{array}$ & $\begin{array}{c}\text { 位置 } \\
\text { location }\end{array}$ & $\begin{array}{l}\text { CR 长度 } \\
\text { CRLength }\end{array}$ \\
\hline 大鳄龟 & Macroclemys temminckii & EF071948 & TA & 20 & $1047-1087$ & 1089 \\
\hline 小鳄龟 & Chelydra serpentina & EF122793 & $\begin{array}{l}\text { TA } \\
\text { TATAT }\end{array}$ & $\begin{array}{l}17 \\
12\end{array}$ & $1026-1124$ & 1124 \\
\hline 平胸龟 & Platysternon megacephalum & DQ016387 & $\begin{array}{l}\text { AGTATGTTAT } \\
(\mathrm{GTT})_{2}(\mathrm{TA})_{2} \text { ACATAT }\end{array}$ & $\begin{array}{c}4 \\
13\end{array}$ & $831-1119$ & 1119 \\
\hline 乌龟 & Chinemys reevesii & AY676201 & $(\mathrm{TA})_{2} \mathrm{TCA}$ & 10 & $916-986$ & 1072 \\
\hline 金头闭壳龟 & Cuora aurocapitata & AY874540 & $(\mathrm{TA})_{2} \mathrm{TTAT}$ & 48 & 896-1368 & 1378 \\
\hline 锦龟 & Chrysemys picta & NC_002073 & $(\mathrm{TA})_{2} \mathrm{~T}$ & 23 & $982-1097$ & 1179 \\
\hline 中华鳖 & Pelodiscus sinensis & AY687385 & ACATAC & 33 & $1019-1217$ & 1829 \\
\hline 缅甸陆龟 & Indotestudo elongata & DQ656607 & $(\mathrm{CAA})_{2} \mathrm{ACTTA}$ & 2 & $647-669$ & 1293 \\
\hline 㲕动胸龟 & Kinosternon odoratus & AF316138 & TA & 16 & $961-993$ & 1145 \\
\hline
\end{tabular}

相关序列、保守序列 F 和保守序列 CSB-1、CSB-2 和 CSB-3, 并认为这些单元行使重要的功能, 受强 烈的自然选择作用。本文通过比较大鳄龟、小鳄龟 和平胸龟 mtDNA CR 区序列, 成功的找到了 3 种龟 类 mtDNA CR 区上的主要功能单元一终止相关序 列、保守序列 F 和保守序列 CSB-1、CSB-2 和 CSB-3。 在进行龟鳖类 mtDNA 控制区研究中, 这些单元有 着重要的参考价值。

一般认为动物 mtDNA CR 区的终止序列区是最 容易发生变异的区域（Tang et al，2005）。本研究 中, 三种龟 mtDNA CR 区终止序列区的长度范围为 280-338 bp, 差异明显。因此, 终止序列区的序列 可以为将来进行物种的种群分化研究和资源保护 研究提供基础。

Walbarg et al(1981)认为哺乳类动物 mtDNA CR 区保守序列区包括 3 个 CSB 区, 它们之间的距离随 物种的不同而变化, 且这三个区段之间容易发生短 或长的重复序列的插入。本研究中, 三种龟 mtDNA
CR 区 CSB1-3 区段之间的长度为 $114-128 \mathrm{bp}$ ，长 度相差不大, 并没有发生重复序列的插入, 可见龟 鳖类动物和哺乳类动物的 mtDNA 控制区 CSB1-3 区存在差异, 可能与龟鳖类动物线粒体进化速率较 慢, 具有较高的保守性有关。

$\mathrm{Su}$ （2005）曾报道不同的物种 mtDNA CR 区重 复序列不同, 重复序列所在的位置及重复序列基序 (motif) 也有不同, 这种差异在个体间表现为 mtDNA 分子的长度变异 (molecule size variation), 并认为 $\mathrm{CR}$ 区长度的变异主要是由重复序列的差异 而造成的。本研究中 9 种龟鳖类动物 mtDNA CR 区 中微卫星序列所在位置、微卫星基序和重复次数各 不相同, 差异明显。最长的微卫星序列长度为金头 闭壳龟 $384 \mathrm{bp}$, 其 CR 区长度为 $1378 \mathrm{bp}$, 低于中华 鳖的控制区长度。可以看出微卫星序列是影响控制 区长度的原因之一, 并不是长度变异的主要原因。

Zhang et al（2000）曾报道不同鲟鱼类线粒体微 卫星序列在鲟鱼类的进化过程中扮演着重要角色, 
其碱基差异大小与它们亲缘关系的远近成正比关 系。国内外许多学者都对平胸龟和鳄龟的分子系统 学进行过研究, 观点不一。Wu et al (1999) 根据 12S rRNA 序列构建的系统发生树建议将平胸龟并到鳄 龟科, 成为鳄龟科的一个属。Cervelli et al（2003） 根据核基因 (U17 snoRNA) 序列构建的系统树认为 平胸龟不应并到鳄龟科, 而应该成为单独的一个 科。Parham et al（2006）基于大片段的 mtDNA 序 列构建系统发树也认为平胸龟应为一个有效科。本 研究中, 9 种龟鳖类 mtDNA CR 区都存在着类似的 微卫星序列, 其中大鳄龟、小鳄龟和㞗动胸龟微卫 星序列以 $\mathrm{TA}$ 重复为主, 只在重复次数上有所差别, 其碱基差异相对较小, 可见鳄龟科和动胸龟科的亲 缘关系可能较近。乌龟和金头闭壳龟微卫星基序 之间的碱基差异仅为 1 个（见表 2)，乌龟和锦龟

\section{参考文献:}

Cervelli M, Oliverio M, Bellini A, Bologna M, Cecconi F and Mariottini P. 2003. Structural and sequence evolution of U17 small nucleolar RNA (snoRNA) and its phylogenetic congruence in chelonians [J]. $\mathrm{J} \mathrm{Mol}$ Evol, 57: 73-84.

Fu YY, Gu WW, Liu YZ, Peng JY, Chang H. 2006. Genetic analysis of the polymorphism of mtDNA D-loop and microsatellite loci in Tibet mini-pigs[J]. Acta laboratoriumanimalis scientia sinica, 14(4): 318-321. [付艳艳, 顾为望, 刘运忠, 彭瑾瑜, 常 弘. 2006. 西藏 小型猪线粒体 D-loop 区及微卫星多态性的遗传学分析. 中国实验 动物学报, 14(4): 318-321.]

Guo XH, Liu SJ, Liu Q, Liu Y. 2004. New progresses on mitochondrial DNA in fish [J]. Acta Genetica Sinica. 31(9): 983-1000. [郭新红, 刘 少军, 刘 巧, 刘 笏. 2004. 鱼类线粒体 DNA 研究新进展. 遗传 学报, 31(9): 983-1000.]

Hackler JC, Van den Bussche RA, Leslie DM. 2007. Characterization of microsatellite DNA markers for the alligator snapping turtle, Macrochelys temminckii [J]. Molecular Ecology Notes, 7: 474-476.

Hell TA. 1999. Bioedit A user-friendly biological sequence alignment editor and analysis program for windows 95/98/NT[J]. Nucleic Acids Sym positon Series, 41: 95-98.

Inoue JG, Miya M, Katsumi T, Nishida M. 2003. Evolution of the deep-sea gulper eel mitochondrial genomes: large-scale gene rearrangements originated within the Eels[J]. Mol. Biol. Evol, 20(11): 1917-1924.

Krenz JG, Naylor GJP, Shaffer HB, Jansen, FJ. 2005. Molecular phylogenetics and evolution of turtles [J]. Mol Phylog Evol, 37: 178-191.

Kumar S, Tamura k, Nei M. 2004. An integrated software for molecular evolutionary genetics analysis and sequence alignment[J]. Brief Bioinformatics, 5(2): 150-163.

Kumazawa Y, Ota H, Nishida M. 1998. The complete nucleotide sequence of a snake (Dinodon semicarinatus) mitochondrial genome with two identical control regions [J]. Genetics, 150(1): 313-329.

Liu H, Yang G, Wei FW, Li M, Hu JC. 2003. Sequence variability of the mitochondrial DNA control region and population genetic structure of sika deers (Cervus nippon) in China [J]. Acta Zool Sinica. 49(1): 53-60. [刘 海, 杨 光, 魏辅文, 李 明, 胡锦且. 2003. 中国大陆梅花
之间的差异为 2 个, 金头闭壳龟与锦龟之间的差异 为 3 个，可见乌龟和金头闭壳龟的亲缘关系较近。 平胸龟与大鳄龟和小鳄龟的微卫星序列差异较大, 可见其亲缘关系较远。此结果支持平胸龟应为一个 单独的科。

Zardoya \& Meyer（1998）曾报道非洲侧颈龟 mtDNA 控制区中存在微卫星序列, 并认为微卫星 序列可以作为种群遗传的一种新型分子标记。根据 国外已报道的有限资料（Stuart et al，2004）以及本 文的研究结果, 可以推测龟鳖类动物 mtDNA CR 区 中可能广泛存在着微卫星序列。在以后的研究中, 可以利用龟鳖类各个科属 mtDNA 控制区存在的差 异设计特异引物, 以进一步利用 mtDNA 微卫星这 一新型高效的分子标记对动物资源进行研究。

鹿 mtDNA 控制区序列变异及种群遗传结构分析. 动物学报, 49(1): 53-60.]

Liu HZ. 2002. The structure and evolution of the mtDNA control region in fish: taking example for Acheilognathinae [J]. Progress in Natural Sciences, 12(3): 266-270. [刘焕章. 2002. 鱼类线粒体 DNA 控制区的 结构与进化: 以鳑鲅鱼类为例.自然科学进展, 12(3): 266-270.]

Maurol DS, Gower DJ, Wilkinson M, Zardoyal R. 2005. A hotspot of gene order rearrangement by tandem duplication and random loss in the vertebrate mitochondrial genome[J]. Mol. Biol. Evol, 23: 227-234.

Parham JF, Feldman CR, Boore JL. 2006. The complete mitochondrial genome of the enigmatic bigheaded turtle (Platysternon): Description of unusual genomic features and the reconciliation of phylogenetic hypotheses based on mitochondrial and nuclear DNA [J]. BMC Evolutionary Biology, 6: 11-25.

Peng QL, Pu YG, Nie LW. 2006. Complete mitochondrial genome of Chinese big-headed turtle, Platysternon megacephalum, with a novel gene organization in vertebrate mtDNA[J]. Gene, 380: 14-20.

Randi E, Lucchini V. 1998. Organization and evolution of the mitochondrial DNA control region in the avian genus Alectoris[J]. Mol Evol, 47(4): 449-462.

Sambrock J, David WR. 2001. Molecular Cloning: A Laboratory Mannual [M], 3rd edn. New York: Cold Spring.

Sbisa E, Tanzariello F, Reyes A, Pesole G, Saccone C. 1997. Mammalian mitochondrial D-loop region structural analysis: identification of new conserved sequences and their functional and evolutionary implications [J]. Gene, 205(1-2): 125-140.

Serb JM, Phillips CA, Iverson JB. 2001. Molecular Phylogeny and Biogeography of Kinosternon flavescens based on complete mitochondrial control region sequences[J]. Molecular Phylogenetics and Evolution. 18(1): 149-162.

$\mathrm{Su}$ Y. 2005. Conserved and heteroplasmy on mitochondrial DNA control region in Animal [J]. Sichuan J zool, 24(4): 669-671. [苏 瑛. 2005. 动 物 mtDNA 控制区及保守与异质. 四川动物, 24(4): 669-671.]

Stuart BL, Parham JF. 2004. Molecular phylogeny of critically endangered Indochinese box turtle (Cuora galbinifons) [J]. Molecular phylogenetics and evolution, 31: 164-177. 
Tang QY, Liu HZ, Yang XP, Xiong BX. 2005. Studies on the structure of the mitochondrial DNA control region and phylogenetic relationships of the subfamily botiinae [J]. Acta Hydrobiological Sinica. 29(6): 645-653. [唐琼英, 刘焕章, 杨秀平, 熊邦喜. 2005. 沙鳅亚科鱼类 线粒体DNA控制区结构分析及系统发育关系的研究. 水生生物学 报, 29(6): 645-653.]

Thompson JD, Higgins DS, Gibson TJ. 1994. CLUSTAL W: Improving the sensitivity of progressive multiple sequence alignment through sequence weighting, postions-specific gap penalties and weight matrix choice[J]. Nucleic Acids Res, 22: 4673-4860.

Walberg MW, Clayton DA. 1981. Sequence and propertiesof the human KB cell and mouse L cell D-loop regions of mitochondrial DNA [J]. Nucleic Acids Res, 9(20): 5411-5421.

Wu P, Zhou KY, Yang Q. 1999. Phylogeny of Asian freshwater and terrestrial turtles based on sequence analysis of $12 S$ rRNA gene fragment [J]. Acta Zool Sin, 45(3): 260-267. [吴 平, 周开亚, 杨 群. 1999. 亚洲淡水和陆生龟鳖类 $12 S$ rRNA 基因片段的序列分析和系 统发生研究. 动物学报, 45(3): 260-267.]

Zardoya R, Meyer A. 1998. Cloning and characterization of a microsatellite in the mitochondrial control region of the African side-necked turtles, Pelomedusa subrufa [J]. Gene, 216: 149-153.
Zardoya R, Trillo EM, Veith M, Meyer A. 2003. Complete nucleotide sequence of the mitochondrial genome of a salamander, Mertensiella luschani[J]. Gene, 317: 17-27.

Zeng QL, Liu HZ. 2001. Study on mitochondrial DNA control region of the Ictiobus cyprinellus[J]. Journal of Hubei University, 23(3): 261-264. [曾青兰, 刘焕章. 2001. 大口胭脂鱼线粒体 DNA 控制区序 列的研究. 湖北大学学报, 23(3): 261-264.]

Zhang P, Zhou H, Liang D, Liu YF, Chen QY, Qu LH.. 2005. The complete mitochondrial genome of a tree frog, Polypedates megacephalus (Amphibia: Anura: Rhacophoridae), and a novel gene organization in living amphibians[J]. Gene, 346: 133-143.

Zhang SM, Wu QJ, Zhang YP. 2000. Tandem repeats of Chinese sturgeon (acipenser sinensis) and related species and its significance in evolution [J]. Chin J Biochem Mol Biol, 16(4): 458-461. [张四明, 吴清 江, 张亚平. 2000. 中华鲟及相关种类的mtDNA控制区串联重复序 列及其进化意义. 中国生物化学与分子生物学报, 16(4): 458-461.]

Zhang Y, Zhang E, He SP. 2003. Studies on the structure of the control region of the bagridae in China and its phylogentic significance[J]. Acta Hydrobiological Sinica, 27(5): 463-467. [张 燕, 张 鸮, 何舜 平. 2003. 中国魭科鱼类线粒体 DNA 控制区结构及其系统发育分析. 水生生物学报, 27(5): 463-467.] 